\title{
Resección laparoscópica en cáncer de recto etapa III: ¿mejor de lo que esperábamos?*
}

\author{
Drs. JOSÉ GELLONA V. ${ }^{1}$, GONZALO URREJOLA S. ${ }^{1}$, FELIPE QUEZADA D. ${ }^{1}$, RODRIGO MIGUIELES C. ${ }^{1}, \mathrm{M}$. \\ ELENA MOLINA P. ${ }^{1}$, FELIPE BELLOLIO R. ${ }^{1}$, ÁLVARO ZÚÑIGA D. ${ }^{1}$
}

1 Departamento de Cirugía Digestiva. Facultad de Medicina. Pontificia Universidad Católica de Chile. Santiago, Chile.

\begin{abstract}
\section{Laparoscopic Resection in Stage III Rectal Cancer: Better than we expected?}

Introduction: Laparoscopic approach to management of rectal cancer (CR) has shown similar results compared to conventional technique. In some series, patients in stage III showed a better survival function in laparoscopic approach. The aim of this study is to compare disease specific survival (SE) and disease free survival (SLE) in patients with stage III rectal cancer treated with radical surgery via laparoscopy or laparotomy. Method: Historic cohort study, all stage III patients treated with elective radical surgery for CR in the period between August 2005 and May 2012 were included. Demographic, surgical specimens and survivor function for SE and SLE were compared. Results: A total of 51 patients were included, 29 laparoscopic and 22 open. The groups were similar in demographic data. Number of lymph nodes, compromised lymph nodes and distal margin distance was significantly higher in the open approach. The percentage of low lymph node count was $9.1 \%$ in the open group and $34.5 \%$ in the laparoscopic group $(\mathrm{p}=0.03)$. SE estimated at 5 years was $50 \%$ and $80 \%$ in the open and laparoscopic approach respectively $(\mathrm{p}=0.019)$. On multivariate analysis, laparoscopic approach was an independent factor for better SE and SLE. Tumor size was an independent risk factor for poor SLE. Conclusion: In our series, laparoscopic approach would be and independent factor for better survival in patients with stage III CR. Tumor size would be associated with poor SLE.
\end{abstract}

Key words: Rectal cancer, stage, survival, laparoscopy.

\section{Resumen}

Introducción: El abordaje laparoscópico del cáncer de recto (CR) ha mostrado resultados similares al abordaje tradicional. Incluso, el subgrupo de pacientes en etapa III tendría ventajas en sobrevida al comparar con el abordaje clásico. El objetivo de este estudio es evaluar la función de sobrevida específica (SE) y libre de enfermedad (SLE) en pacientes tratados quirúrgicamente por cáncer de recto etapa III y comparar según la vía de abordaje. Método: Estudio de cohorte histórica, se incluyeron pacientes sometidos a cirugía radical curativa electiva por CR, etapa III entre agosto de 2005 y mayo de 2012. Se compararon variables demográficas, de la pieza quirúrgica y las funciones de SE y SLE. Resultados: Serie compuesta por 51 pacientes,

*Recibido el 8 de octubre de 2012 y aceptado para publicación el 22 de noviembre de 2012.

Los autores no tienen conflicto de interés ni fuentes de financiamiento que reportar.

Correspondencia: Dr. José Gellona V.

Marcoleta 350, Santiago, Chile.

jgellona@yahoo.com 
29 abordados por laparoscopia y 22 por vía tradicional. Comparables en cuanto a variables demográficas. El número de ganglios obtenidos, comprometidos y distancia al margen distal fueron significativamente mayores en el grupo abierto. El porcentaje de cosecha ganglionar insuficiente fue de $9,1 \%$ en el grupo abierto y $34,5 \%$ en los laparoscópicos $(\mathrm{p}=0,03)$. La SE a 5 años fue de $50 \%$ para los abiertos y $80 \%$ para el grupo laparoscópico $(\mathrm{p}=0,019)$. El análisis multivariado mostró a la laparoscopia como factor independiente de mejor SE y SLE. El tamaño tumoral se comportó como factor de riesgo para menor SLE. Conclusión: En este estudio, el abordaje laparoscópico sería un factor de mejor SE y SLE en pacientes con CR etapa III. El tamaño tumoral sería un factor de menor SLE.

Palabras clave: Cáncer de recto, etapificación sobrevida, laparoscopia.

\section{Introducción}

En el tratamiento quirúrgico del cáncer de recto (CR), el abordaje laparoscópico no tuvo una aceptación inmediata debido a los resultados de la primera serie publicada, que mostraron una mayor frecuencia de compromiso del margen circunferencial en el estudio de la pieza quirúrgica ${ }^{1}$. Sin embargo, ante la evidencia acumulada, se ha llegado a aceptar que en manos expertas se puede realizar la misma calidad de cirugía, con menor morbilidad y similares resultados en sobrevida global y libre de enfermedad ${ }^{2}$. Incluso en etapa III de la enfermedad se ha observado una ventaja para el abordaje laparoscópico, en al menos dos series de cáncer de recto ${ }^{3,4}$. No hay una razón específica que explique estos hallazgos, pero se ha postulado que la laparoscopia, por un menor stress quirúrgico, altera en menor medida la respuesta inmune, la cual tiene un rol importante en la progresión tumoral y la diseminación a distancia $^{5-10}$. Sin embargo, los estudios son longitudinales comparativos, y no analizan en detalle el subgrupo de pacientes en etapa III, por lo que no está claro si se seleccionaron pacientes distintos para la técnica laparoscópica o abierta. Además, para atribuir estos hallazgos a lo mencionado anteriormente se debe comprobar que la cirugía laparoscópica cumple las mismas exigencias técnicas que el abordaje tradicional, con la confirmación anatomopatológica de que las piezas operatorias no son distintas en cuanto a márgenes, cosecha ganglionar e indemnidad del mesorrecto. El objetivo de este estudio es analizar la función de sobrevida específica (SE) y libre de enfermedad (SLE) en una serie de pacientes con cáncer de recto en etapa III sometidos a resección radical por vía laparoscópica o abierta, y mediante un modelo de regresión ajustar por los posibles factores confundentes.

\section{Método}

\section{Diseño del estudio y pacientes}

Se realizó un estudio de cohorte histórica, analizando el subgrupo de pacientes con $\mathrm{CR}$ en etapa
III sometidos a resección radical (resección anterior o resección abdominoperineal) curativa y electiva, entre agosto de 2005 y mayo de 2012. Los datos se obtuvieron de la base de datos de neoplasia colorrectal mantenida de manera prospectiva, de la revisión de registros clínicos y anatomopatológicos, y mediante entrevista individual. Se consideró como $\mathrm{CR}$ a los adenocarcinomas ubicados hasta $15 \mathrm{~cm}$ proximal al margen anal. Se subdividieron según localización, tercio superior (11 y 15$)$ y tercio medio o inferior $(0-11 \mathrm{~cm})$. Se consignaron los datos demográficos, localización del tumor, IMC, ASA, uso de neoadyuvancia, tamaño tumoral, compromiso de márgenes y recuento ganglionar. Los pacientes con tumores del tercio medio o inferior T3, T4 o con adenopatías fueron sometidos a radio-quimioterapia neo adyuvante (45 Gy a la pelvis más 5 Gy de refuerzo al tumor, junto con $5 \mathrm{FU}$ en infusión asociado a leucovorina durante un período de 6 semanas). Algunos tumores de tercio superior T3 voluminosos o T4 que estaban en el límite entre tercio superior y el tercio medio, también fueron sometidos a neoadyuvancia. La cirugía fue realizada entre 6 a 10 semanas luego de finalizada la neoadyuvancia. Todos los pacientes fueron operados por cirujanos colorrectales o residentes de la especialidad bajo supervisión directa. La técnica quirúrgica, independiente del abordaje (abierto o laparoscópico), incluyó la resección total del mesorrecto y ligadura de los vasos mesentéricos en su origen. En los casos de resección anterior, la anastomosis colorrectal se practicó con doble grapadura. En algunos casos de resecciones anteriores bajas se realizó anastomosis colo-anal manual por vía anal. El uso de ileostomía de protección se indicó según criterio del cirujano. En todos los pacientes se dejó un drenaje tubular en la pelvis extraído por contrabertura en la pared abdominal. El estudio anátomo patológico de la pieza operatoria se realizó en base a los criterios de la clasificación TNM.

Se definió como cosecha ganglionar insuficiente a la obtención de menos de 12 ganglios en la pieza operatoria. El compromiso de margen radial fue definido como presencia de células tumorales a $1 \mathrm{~mm}$ o menos de dicho margen. 


\section{Análisis estadístico}

Se analizaron y compararon variables demográficas y de la pieza quirúrgica. Las variables cualitativas se analizaron mediante pruebas de asociación $\left(\chi^{2}\right.$ y Fisher según corresponda) y las variables continuas se compararon mediante $\mathrm{t}$ de Student o Man Whitney dependiendo de su distribución. Se confeccionó la función de SE y SLE con el método de Kaplan-Meier y se comparó las curvas con el método de Log-Rank. El análisis uni y multivariado de factores de sobrevida se realizó mediante el modelo proporcional de Cox. Se incluyeron en el análisis multivariado los factores que resultaron significativos en el análisis univariado. Se consideró estadísticamente significativo un $\mathrm{p}<0,05$. El análisis realizado fue por intención de tratamiento.

\section{Resultados}

\section{Descripción de la serie y resultados quirúrgicos}

De un total de 154 pacientes operados por $\mathrm{CR}$ en el período mencionado, se identificaron 51 pacientes en Etapa III, 22 abordados por laparotomía y 29 por laparoscopia. $54,6 \%$ de los pacientes en el grupo abierto de sexo masculino, $72,4 \%$ en el grupo laparoscópico. No se observaron diferencias significativas en cuanto a edad, género, índice de masa corporal (IMC), localización o clasificación ASA (Tabla 1). Con respecto a los resultados quirúrgicos, el tiempo operatorio fue de $120 \mathrm{~min}$ (80-240) en el grupo abierto, mientras que en el grupo laparoscópico fue de $210 \min (130-500), p=0,03$. La estadía postoperatoria fue de 8 (6-14) y 6 (4-50) días para los grupos abierto y laparoscópico respectivamente $(p=0,002)$. Del total de la serie, 5 pacientes presentaron alguna complicación, 2 (9\%) en el grupo abierto y 3 $(10 \%)$ en el grupo laparoscópico $(\mathrm{p}=$ $0,881)$. No se observó mortalidad. Un paciente en el grupo laparoscópico fue convertido a cirugía tradicional por dificultad anatómica.

\section{Anatomía patológica}

Con respecto a la pieza quirúrgica, se observó una tendencia no significativa a resecar tumores de mayor tamaño por vía abierta (Tabla 1). El recuento ganglionar, número de ganglios comprometidos y la distancia al margen distal fueron significativamente mayores en el grupo abierto (Tabla 1). Por el contrario, en el grupo laparoscópico se observó una mayor proporción de pacientes con cosecha ganglionar insuficiente. En el grupo abierto, el 100\% de los pacientes con cosecha ganglionar insuficiente habían recibido neoadyuvancia, en el grupo laparoscópico, el $60 \%$ de las cosechas ganglionares insuficientes recibió neoadyuvancia. La proporción de pacientes con compromiso de margen radial no tuvo diferencias entre ambos grupos (Tabla 1$)$. Hubo $2(3,9 \%)$ pacientes que presentaron el margen distal comprometido, uno en cada grupo.

Tabla 1. Descripción de las características basales de los grupos y los resultados de la pieza quirúrgica

\begin{tabular}{|c|c|c|c|}
\hline Variable & $\begin{array}{l}\text { Abierto } \\
(\mathrm{N}=22)\end{array}$ & $\begin{array}{c}\text { Laparos- } \\
\text { cópico } \\
(\mathrm{N}=29)\end{array}$ & $\mathbf{p}$ \\
\hline Edad (años) & $60,3 \pm 15,9$ & $64,8 \pm 11,4$ & $0,125^{1}$ \\
\hline Sexo (masculino) & $54,6 \%$ & $72,4 \%$ & $0,186^{3}$ \\
\hline IMC & $27,8 \pm 5$ & $25,3 \pm 4,6$ & $0,380^{2}$ \\
\hline Localización tumoral (\%) & & & $0,427^{3}$ \\
\hline Tercio superior & 45,4 & 34,5 & \\
\hline Tercio medio o inferior & 54,6 & 65,5 & \\
\hline Tipo de cirugía (\%) & & & $0,268^{3}$ \\
\hline Resección anterior & 90,9 & 75,9 & \\
\hline Resección abdominoperineal & 9,1 & 24,1 & \\
\hline ASA $(\%)$ & & & 0,509 \\
\hline 1 & 52,4 & 42,9 & \\
\hline 2 & 47,6 & 57,1 & \\
\hline Neoadyuvancia (\%) & 27,3 & 41,4 & $0,296^{3}$ \\
\hline Longitud pieza quirúrgica (cm) & $33,8 \pm 14$ & $30 \pm 9$ & $0,196^{2}$ \\
\hline Tamaño tumoral (cm) & $4,9 \pm 2,7$ & $3,9 \pm 1,8$ & $0,06^{1}$ \\
\hline Número de ganglios obtenidos & $19,5 \pm 10$ & $15 \pm 9$ & $0,01^{2}$ \\
\hline Número de ganglios comprometidos & $3,5 \pm 5$ & $1 \pm 2$ & $0,003^{2}$ \\
\hline Índice ganglionar & $\begin{array}{c}0,16 \\
(0-0,85)\end{array}$ & $\begin{array}{c}0,1 \\
(0-0,6)\end{array}$ & $0,08^{4}$ \\
\hline Distancia al margen distal (mm) & $2 \pm 2$ & $1 \pm 2$ & $0,018^{2}$ \\
\hline Cosecha ganglionar insuficiente (\%) & 9,1 & 34,5 & $0,03^{3}$ \\
\hline Compromiso margen radial (\%) & 9,1 & 6,9 & $0,776^{3}$ \\
\hline
\end{tabular}

${ }^{1}$ Prueba $t$ de Student para muestras independientes, resultados de variable expresados en promedio \pm desviación estándar. ${ }^{2}$ Prueba de Man Whitney, resultados de variable expresados en mediana \pm rango interquartílico. ${ }^{3}$ Prueba de $\chi^{2} .{ }^{4}$ Prueba de Man Whitney, resultados de variable expresados en mediana (mínimo-máximo). 
Tabla 2. Regresión de Cox para las variables significativas incluidas en el modelo

\begin{tabular}{|c|c|c|c|c|}
\hline \multirow[t]{2}{*}{ Variable } & \multicolumn{2}{|c|}{ Sobrevida específica } & \multicolumn{2}{|c|}{ S. Libre de enfermedad } \\
\hline & Hazard Ratio & IC $95 \%$ & Hazard Ratio & IC $95 \%$ \\
\hline Abordaje laparoscópico & 0,106 & $0,018-0,702$ & 0,116 & $0,021-0,652$ \\
\hline Tamaño tumor & 1,208 & $0,862-1,695$ & 1,407 & $1,032-1,918$ \\
\hline Distancia al margen distal & 1,001 & $0,944-1,061$ & 0,947 & $0,896-1,002$ \\
\hline Cosecha ganglionar insuficiente & 1,210 & $0,095-15,299$ & 1,192 & $0,125-11,095$ \\
\hline Longitud de la pieza & 1,013 & $0,960-1,069$ & 1,021 & $0,969-1,076$ \\
\hline $\mathrm{N}^{\circ}$ de ganglios obtenidos & 0,992 & $0,881-1,117$ & 0,998 & $0,891-1,118$ \\
\hline $\mathrm{N}^{\circ}$ ganglios comprometidos & 0,898 & $0,612-1,318$ & 0.796 & $0,560-1,131$ \\
\hline
\end{tabular}

\section{Sobrevida}

Con una mediana de seguimiento para toda la serie de 35 meses, 31,2 en el grupo abierto y 37,8 en el laparoscópico ( $\mathrm{p}=$ 0,338 ), la estimación de SE a 5 años para el grupo abierto fue de $50 \%$ y para el grupo laparoscópico fue de $80 \%(\mathrm{p}=0,019)$, (Figura 1). En SLE, también se observó una diferencia significativa a favor del abordaje laparoscópico (Figura 2).

Del total de la serie, $14(27,5 \%)$ pacientes presentaron progresión de la enfermedad. Dos $(3,9 \%)$ de ellos, abordados por vía abierta, tuvieron una recidiva local, a los 10 y 20 meses de seguimiento. Ambos pacientes fallecieron a los 37 y 27 meses respectivamente. En el resto de los pacientes la progresión fue a distancia, en 5 de ellos hepática, en 4 pulmonar y 3 en ambas localizaciones.

Se realizó un modelo de regresión de proporcional de Cox ajustado por las variables que resultaron significativas en el análisis univariado, tanto para SE como para SLE. Se observó que el abordaje laparoscópico es un factor independiente de mejor SE y SLE, mientras que el tamaño tumoral es un factor de riesgo para menor SLE (Tabla 2). El resto de las variables analizadas no resultaron significativas.

\section{Discusión}

Los resultados observados en esta serie muestran función de SE favorable en pacientes con cáncer de recto etapa III abordados por laparoscopia, que al ajustarla por los posibles confundentes se mantuvo significativa. Este efecto también se ob-

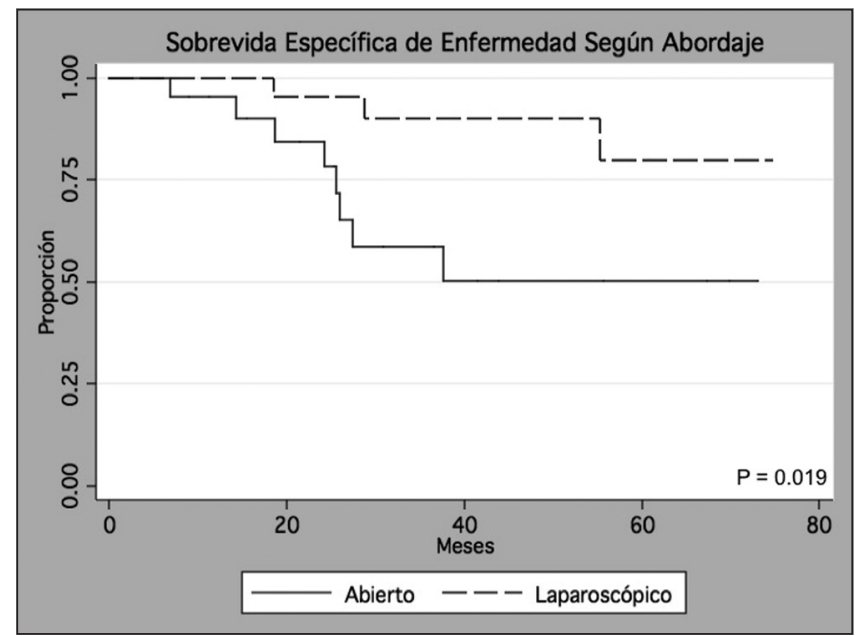

Figura 1. Curva de función de sobrevida específica para cáncer de recto etapa III según abordaje.

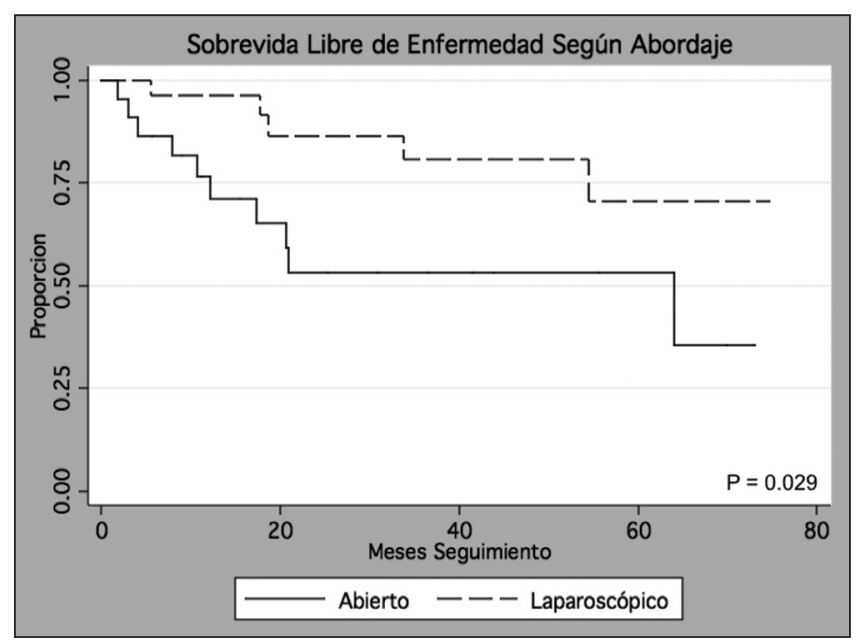

Figura 2. Curva de función de sobrevida libre de enfermedad según abordaje. 
serva al analizar la curva de SLE. Estos hallazgos concuerdan con otras series publicadas tanto para cáncer de colon ${ }^{11}$ como también para cáncer de recto $^{3-4}$. En el estudio de Lacy et al ${ }^{11}$, se observó mejor SE y SLE pero no sobrevida global. Laurent et $\mathrm{al}^{5}$, demostraron una mejor sobrevida global en estos pacientes, sin embargo, en SE y SLE no encontraron diferencias. Morino et $\mathrm{al}^{3}$, observaron estos mismos resultados en sobrevida global y SLE, sin embargo, no evaluaron SE. La curva de sobrevida en el abordaje laparoscópico incluso llega a asemejarse a la de los pacientes etapa $\mathrm{II}^{3-4}$. Es difícil encontrar una razón para explicar este fenómeno, lo primero que se debe descartar es que exista sesgo de selección en este grupo de pacientes. En este estudio, al menos en edad, ASA, IMC y localización del tumor, los grupos fueron comparables.

Con respecto a la pieza operatoria, se observó diferencias en cuanto a tamaño tumoral (no significativa), número de ganglios obtenidos, ganglios comprometidos, distancia al margen distal y cosecha ganglionar insuficiente. El tamaño tumoral podría ser otro factor de sesgo, al distribuirse los tumores más grandes con mayor frecuencia para el grupo abierto. Asimismo, el número de ganglios comprometidos resultó mayor en el grupo abierto, sin embargo, el índice ganglionar no mostró diferencias significativas. Por otro lado, en el grupo laparoscópico se observó mayor porcentaje de cosecha ganglionar insuficiente, menor distancia al margen distal y menor número de ganglios obtenidos, lo que en teoría serían factores de mal pronóstico. El rol de la cosecha ganglionar insuficiente como factor de mal pronóstico en cáncer de recto es debatido y no se ha logrado asociar a peor sobrevida. Es así como Govindarajanet $\mathrm{al}^{12}$, encontraron que un recuento menor a 12 ganglios en pacientes con neoadyuvancia no fue relevante en la sobrevida ni se asoció a sub-etapificación. En el modelo de Cox realizado en este trabajo, la cosecha ganglionar insuficiente no fue un factor de peor SE y SLE al ajustarlo por los otros posibles confundentes. Estos hallazgos hacen posible la opción de que las diferencias en sobrevida se deban a factores desconocidos, más que a los anteriormente expuestos.

La cirugía laparoscópica podría tener una influencia positiva en la respuesta inmune, la cual tiene un rol importante en la diseminación local y a distancia ${ }^{7}$. Se ha observado que en cirugía laparoscópica hay una menor respuesta de fase aguda, mediada principalmente por IL-6 y proteína C reactiva (PCR) como efector final, comparada con la cirugía tradicional ${ }^{7}$. Delgado et al, demostraron esto específicamente en cirugía de colon ${ }^{8}$. Además, la cirugía abierta tendría una mayor depresión de la respuesta inmune celular y alteraría en mayor medi- da la función de los polimorfonucleares, funciones importantes para evitar la diseminación de células neoplásicas y crecimiento tumoral, que probablemente adquiere mayor importancia en etapa III, donde el tumor escapa de lo local ${ }^{7-10}$.

En nuestro estudio, el tamaño tumoral resultó un factor de riesgo para menor sobrevida libre de enfermedad. Poritz et $\mathrm{al}^{13}$, analizaron el tamaño tumoral (entre otras variables) y encontraron una asociación con menor sobrevida en pacientes con cáncer colorrectal etapa III. Otros estudios han asociado al tamaño tumoral con el mayor riesgo de compromiso de margen circunferencial, resultando un factor indirecto de peor pronóstico ${ }^{14,15}$.

Este estudio tiene ciertas limitaciones, algunas ya mencionadas. La principal es el tamaño de la muestra que puede carecer de poder para demostrar con significancia estadística la influencia de todos los confundentes. Por otro lado, al no ser un estudio aleatorio, no es posible asegurar una distribución pareja de factores no conocidos, potencialmente significativos para los resultados. En conclusión, el presente estudio muestra mejor sobrevida específica y libre de enfermedad en pacientes operados de cáncer de recto en etapa III por vía laparoscópica. Esta mayor sobrevida no se encuentra influenciada por factores analizados del paciente ni de la pieza quirúrgica. Sin embargo, dadas las limitaciones ya señaladas debemos ser cautos en la interpretación de estos resultados.

\section{Referencias}

1. Guillow PJ, Quirke P, Thorpe H, Walker J, Smith A, Heath RM et al. Short-term endpoints of conventional versus laparoscopic-assisted surgery in patients with colorectal cancer (MRC CLASICC trial): multicenter, randomized controlled trial. Lancet 2005;365:1718-26.

2. Siegel R, Cuesta MA, Targarona E, Bader FG, Morino M, Corcelles R, et al. Laparoscopic extraperitoneal rectal cancer surgery: the clinical practice guidelines of the European Association for Endoscopic Surgery (EAES). Surg Endosc. 2011;25:2423-40.

3. Morino M, Allaix ME, Giraudo G, Corno F, Garrone C. Laparoscopic versus open surgery for extraperitoneal rectal cancer. A prospective comparative study. Surg Endosc. 2005;19:1460-7.

4. Laurent C, Leblanc F, Wütrich P, Scheffer M, Rullier E. Laparoscopic versus open surgery for rectal cancer. Long term oncologic results. Ann Surg. 2009;250:54-61.

5. Hansbrough JF, Bender EM, Zapata-Sirvent R, Anderson J. Altered helper and suppressor lymphocyte populations in surgical populations in surgical patients: a measure of postoperative immunosuppression. Am J Surg. 1984;148:303-7.

6. Decker D, Schondorf M, Bidlingmaier F, Hirner A, 
von Ruecker AA. Surgical stress induces a shift in the type-1/type-2 T-helper cell balance, suggesting down regulation of cell mediated and upregulation of antibody-mediated immunity commensurate to the trauma. Surgery 1996;119:316-25.

7. Vittimberga FJ Jr, Foley DP, Meyers WC, Callery MP. Laparoscopic surgery and the systemic immune response. Ann Surg. 1998;227:326-34.

8. Delgado S, Lacy AM, Filella X, Castells A, García-Valdecasas JC, Pique JM, et al. Acute phase response in laparoscopic and conventional colectomy in colon cancer: a randomized study. Dis Colon Rectum 2001;44:638-46.

9. Kuntz C, Wunsch A, Bay F. Prospective randomized study of stress andimmune response after laparoscopic vs conventional colonic resection. Surg Endosc. 1998;12:963-7.

10. Leung KL, Lai PB, Ho RL. Systemic cytokine response after laparoscopic-assisted resection of recto-sigmoid carcinoma: a prospective randomized trial. Ann Surg. 2000;231:506-11.

11. Lacy A, García-Valdecasas JC, Delgado S, Castells
A, Taurá P, Piqué JM, et al. Laparoscopy-assisted colectomy versus open colectomy for treatment of non-metastatic colon cancer: a randomized trial. Lancet 2002;359:2224-9.

12. Govindarajan A, Gönen M, Weiser MR, Shia J, Temple LK, Guillem JG, et al. Challenging the feasibility and clinical significance of current guidelines on lymph node examination in rectal cancer in the era of neoadjuvant therapy. J Clin Oncol . 2011;29:4568-73.

13. Poritz LS, Sehgal R, Hartnett K, Berg A, Koltun WA. Tumor volume and percent positive lymph nodes as a predictor of 5-year survival in colorectal cancer. Surgery 2011;150:649-55.

14. Hiranyakas A, da Silva G, Wexner SD, Ho YH, Allende $\mathrm{D}$, Berho $\mathrm{M}$, et al. Factors Influencing Circumferential Resection Margin in Rectal Cancer. Colorectal Dis. 2012 Jul 10. [Epub ahead of print]. Citado el 12 de julio de 2012. Disponible en www.onlinelibrary.wiley.com.

15. Oh SJ, Shin JY. Risk factors of circumferential resection margin involvement in the patients with extraperitoneal rectal cancer. J Korean Surg Soc. 2012;82:165-71. 